\title{
Genotype-phenotype correlation of renal lesions in the tuberous sclerosis complex
}

Yoshinari Muto $^{1 凶}$, Hitomi Sasaki ${ }^{1,3}$, Makoto Sumitomo ${ }^{1,3}$, Hidehito Inagaki $\mathbb{D}^{2}$, Maki Kato ${ }^{2}$, Takema Kato ${ }^{2}$, Shunsuke Miyai ${ }^{2}$, Hiroki Kurahashi ${ }^{2}$ and Ryoichi Shiroki ${ }^{1}$

(c) The Author(s) 2022

Tuberous sclerosis complex (TSC) is an autosomal dominant disease caused by loss-of-function mutations in either of two tumor suppressor genes, TSC1 and TSC2. These mutations lead to the growth of benign tumors and hamartomas in many organs, including those of the central nervous system, the skin, and the kidneys. To investigate the genotype-phenotype correlation, we performed sequence analysis of the TSC1/2 genes using next-generation sequencing. We classified 30 patients with TSC whose pathogenic variants were identified into two groups: those with mutations producing premature termination codons (PTCs) and those with missense mutations. Then, we compared the phenotypes between the two groups. Patients with a PTC were significantly more likely to manifest the major symptoms of the diagnostic criteria than those without a PTC $(P=0.035)$. The frequencies of subependymal nodules $(P=0.026)$, cortical tubers $(P=0.026)$, and renal cysts $(P=0.026)$ were significantly higher in PTC-containing variants than in cases without a PTC. When the analyses were limited to renal angiomyolipoma (AML) cases with TSC2 mutations, there was no difference in tumor size between cases with and without a PTC. However, the cases with a PTC showed a trend toward disease onset at a younger age and multiple tumors, and bilateral disease was observed in their AML lesions. TSC patients with PTC-producing mutations might potentially manifest more severe TSC phenotypes than those with missense mutations. A larger-scale study with appropriate samples deserves further investigation.

Human Genome Variation (2022) 9:1-5; https://doi.org/10.1038/s41439-022-00181-1

\section{INTRODUCTION}

Tuberous sclerosis complex (TSC) is an autosomal dominant genetic disease affecting one in every $6,000-10,000$ live births ${ }^{1}$. It is mainly characterized by the growth of benign tumors and other hamartoma lesions in various organs throughout the body, including the kidney, brain, lung, skin, and heart ${ }^{2}$. Renal angiomyolipoma (AML) is a pathologically benign mesenchymal kidney tumor characterized by vascular smooth muscle and adipocyte elements ${ }^{3}$. AMLs are rare in the general population (autopsy prevalence of $\sim 1$ in 1,000). However, they affect $>70 \%$ of adults with TSC, where they are usually multifocal and bilateral ${ }^{2}$. In fact, for those with TSC, acute bleeding of renal AML caused by spontaneous or traumatic rupture is the main cause of death ${ }^{4}$. Moreover, pathogenic mutations in TSC1 and TSC2 are found in $75-90 \%$ of cases that meet the standard clinical criteria for $\mathrm{TSC}^{5,6}$. The TSC 1 gene is located at $9 q 34$ and contains 23 exons. It is $50 \mathrm{~kb}$ in length and codes for the hamartin protein (molecular weight, $130 \mathrm{kDa}, 1164$ amino acids). The TSC2 gene is located at $16 \mathrm{p} 13.3$ and contains 42 exons. It is $45 \mathrm{~kb}$ in length and codes for the tuberin protein (molecular weight, $190 \mathrm{kDa}, 1807$ amino acids) ${ }^{7}$. Approximately $60-70 \%$ of TSC cases are sporadic, which reflects a high de novo mutation rate in these genes ${ }^{8}$.

Previous genetic studies have shown that renal AML occurring in TSC cases was caused by the inactivation of both pairs of alleles of either TSC1 or TSC2, which is consistent with Knudson's 2-hit tumor suppressor gene model ${ }^{2,5,9-12}$. TSC1 (hamartin) and TSC2 (tuberin) complexes act as inhibitors of the mTOR pathway ${ }^{10,11,13,14}$. Biallelic mutations of TSC1 or TSC2 activate the mTOR pathway, leading to an advantage for the growth of affected cells. mTOR inhibitors have been demonstrated to be effective in suppressing the growth of TSC tumors $^{14}$.

Pathogenic mutations in the TSC1 or TSC2 genes are often nonsense or frameshift mutations that lead to premature termination codons $(\mathrm{PTCs})^{10}$. When mRNA contains a PTC, cells utilize an mRNA decay pathway, called nonsense-mediated mRNA decay (NMD), to eliminate nonfunctional transcripts ${ }^{15}$. Alleles with nonsense or frameshift mutations do not produce any protein. However, alleles with missense mutations produce proteins with partially altered amino acid sequences that can affect protein stability, hydrogen bonding, dynamics, and activity, leading to alterations in protein function ${ }^{16}$. Thus, it is possible that the type of mutations in the TSC1 or TSC2 gene could affect the clinical phenotypes of the patient. The purpose of this study was to clarify the significance of germline mutations in the characteristics of renal AML in TSC cases.

\section{MATERIALS AND METHODS}

Patients

This study was approved by the local Ethical Review Committee of Fujita Health University (HG-20-054). Written informed consent was obtained

${ }^{1}$ Department of Urology, School of Medicine, Fujita Health University, Toyoake, Japan. ${ }^{2}$ Division of Molecular Genetics, Institute for Comprehensive Medical Science, Fujita Health University, Toyoake, Aichi 470-1192, Japan. ${ }^{3}$ These authors contributed equally: Hitomi Sasaki, Makoto Sumitomo. ${ }^{\circledR}$ email: naru.muto@gmail.com 
Table 1. Phenotypic characteristics of patients with tuberous sclerosis complex in this study.

\begin{tabular}{|c|c|c|c|c|c|}
\hline \multicolumn{2}{|c|}{ Characteristic } & All $(N=30)$ & TSC1 $(N=4)$ & $\operatorname{TSC2}(N=23)$ & NMI $(N=3)$ \\
\hline \multicolumn{2}{|c|}{ Median age, years (range) } & $24(14-28)$ & $21.5(16.3-28)$ & $22(11.3-27.3)$ & $34(30.5-39.5)$ \\
\hline \multirow[t]{2}{*}{ Gender } & Male (\%) & $12(40.0)$ & $1(25.0)$ & $11(47.8)$ & $0(0)$ \\
\hline & Female (\%) & $18(60.0)$ & $3(75.0)$ & $12(52.1)$ & $3(100)$ \\
\hline \multicolumn{2}{|c|}{ Hypomelanotic macules (\%) } & $9(30.0)$ & $2(50.0)$ & $6(26.1)$ & $1(33.3)$ \\
\hline \multicolumn{2}{|c|}{ Angiofibromas (\%) } & $20(66.7)$ & $1(25.0)$ & 17 (73.9) & $2(66.7)$ \\
\hline \multicolumn{2}{|c|}{ Ungual fibromas (\%) } & $9(30.0)$ & $1(25.0)$ & $7(30.4)$ & $1(33.3)$ \\
\hline \multicolumn{2}{|c|}{ Multiple retinal hamartomas (\%) } & $7(23.3)$ & $0(0)$ & $7(30.4)$ & $0(0)$ \\
\hline \multicolumn{2}{|c|}{ Cortical tuber (\%) } & $26(86.7)$ & $4(100)$ & $21(91.3)$ & $1(33.3)$ \\
\hline \multicolumn{2}{|l|}{ SEN (\%) } & $26(86.7)$ & $4(100)$ & $21(91.3)$ & $1(33.3)$ \\
\hline \multicolumn{2}{|c|}{ SEGA (\%) } & $4(13.3)$ & $1(25.0)$ & $3(13.0)$ & $0(0)$ \\
\hline \multicolumn{2}{|c|}{ Cardiac rhabdomyoma (\%) } & $6(20.0)$ & $0(0)$ & $6(26.1)$ & $0(0)$ \\
\hline
\end{tabular}

from all 30 patients with TSC included in this study. These patients were located at Fujita Health University and were clinically diagnosed with TSC mainly by clinical features and imaging evaluation according to the latest diagnostic criteria ${ }^{17}$. The median age of the patients was 24 years (range, 14-28 years). There were 12 male (40\%) and 18 female (60\%) patients (Table 1). The age at onset of AML was the age of the patient when they were first diagnosed with renal AML by computed tomography (CT), and the maximum diameter of the AML was measured on CT.

In the clinical phenotypes of these patients, 26 patients (89.7\%) had renal AML, 26 patients (89.7\%) had subependymal nodules, 27 (91.3\%) had cortical tubers, $4(13.8 \%)$ had subependymal giant cell astrocytomas, and 16 (55.2\%) experienced seizures. Additionally, 11 patients (37.9\%) presented with LAM, and 22 patients (75.9\%) exhibited skin lesions (Table 1).

\section{Mutation analysis}

Peripheral blood samples were collected from each patient, and genomic DNA was isolated from peripheral leukocytes (Qiagen, Frankfurt, Germany). The TSC1 and TSC2 genomic regions were amplified using long-range polymerase chain reaction (PCR). The Nextera XT DNA Library Preparation Kit (Illumina, San Diego, CA, USA) was used to fragment the genomic DNA following the manufacturer's instructions. Then, AMPure XP (Beckman Coulter, Pasadena, CA, USA) was used to purify the library DNA and to remove short library fragments. Amplification with DNA polymerase and sequencing of the DNA samples were performed using Illumina MiSeq. Sequence reads were then mapped to a human reference sequence (RefSeq: NM_003639.3), and the identified sequence variants were confirmed by Sanger sequencing. The pathogenicity of the variants was evaluated by standard criteria ${ }^{18}$.

\section{Phenotype-genotype correlation}

To analyze the effect of genetic mutations on the phenotypic appearance in patients with TSC, splicing site mutations were excluded. The frequency of each phenotype was compared between the patients with mutations producing PTC (nonsense mutation or frameshift mutation) and those with missense mutations.

\section{Statistical analysis}

Continuous variables between the two groups were compared by the Mann-Whitney U-test. Proportions between the two groups were compared by Fisher's test, and the $P$-value was calculated. $P$-values $<$ 0.05 were considered statistically significant. Statistical analysis was conducted using the EZR software package (R version 3.4.1, Saitama
Medical Center/Jichi Medical University, Saitama, Japan) ${ }^{19}$, which is a graphical user interface for R version 3.4.1 (R Foundation for Statistical Computing, Vienna, Austria).

\section{RESULTS}

Of the 30 patients enrolled in this study, 23 had mutations in TSC2, four had mutations in TSC1, and no mutation was found in the remaining three patients (Table 1 ).

Of the four cases with TSC1 mutations, three had nonsense mutations, and the other had a frameshift mutation. Among the 23 cases with TSC2 mutations, five had nonsense mutations, eight had frameshift mutations, six had missense mutations, two had inframe deletions, and two had splice site mutations. All of the mutations in TSC1 and the 13 mutations in TSC2 (56.5\%) resulted in PTC (Fig. 1A). Moreover, no mutation hotspots were observed, and the mutations were located throughout the coding region of the genes (Fig. 1B).

To determine the effect of the type of mutations in TSC1 or TSC2 genes on the clinical course of the patient, we analyzed the correlation between the type of mutation, PTC or missense mutation, and the clinical features of the patients. Patients with a PTC were significantly more likely to manifest the major symptoms of the diagnostic criteria than those without a PTC (Table 2) ${ }^{17}$. Among the clinical features (Table 2), hypomelanotic macules, subependymal nodules (SENs), and cortical tubers were significantly more frequent in cases with a PTC $(69.2 \%$ vs. $100 \%, P=$ 0.026), while all the other features did not show any significant differences between PTC and missense mutations. For the renal phenotype, only the frequency of renal cysts was found to be significantly more frequent in cases with PTC mutations $(15.4 \%$ vs. 58.8, $P=0.026$ ) (Table 3). Indeed, the maximum diameter of AML ranged from $2-8.9 \mathrm{~cm}$ (median $3.6 \mathrm{~cm}$ ), and no difference was observed between the presence and absence of PTC mutations (Table 1 and Table 3). However, the median maximum diameter of TSC1 AML was $1.7 \mathrm{~cm}(0.8-3.7 \mathrm{~cm})$, while that of TSC2 was $3.4 \mathrm{~cm}$ $(2.1-8.9 \mathrm{~cm})$. These results suggest that the differences in the causative genes might affect the clinical features, although the differences were not statistically significant (Table 1). Thus, we analyzed the phenotype-genotype correlation in kidney AML lesions, focusing only on patients with TSC2 mutations. 
A

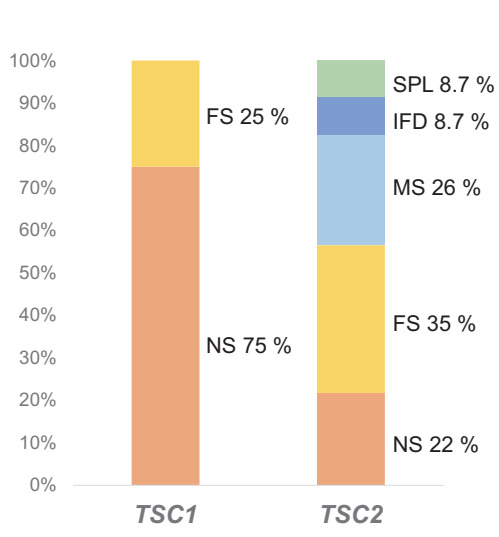

B
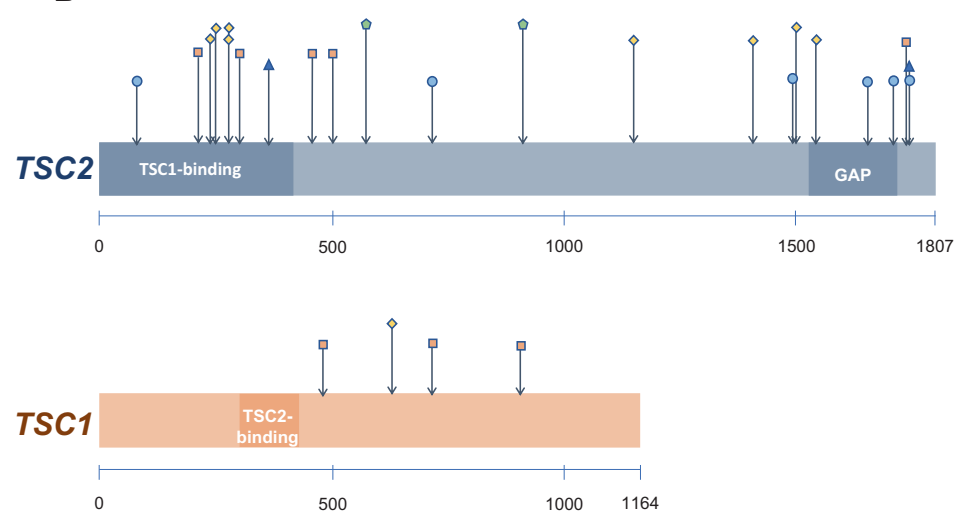

- MS: Missense $\Delta$ IFD: In-frame deletion $\square$ NS: Nonsense $\diamond$ FS: Frames shift $\square$ SPL: Splice shift mutation

Fig. 1 Type and distribution of germline mutations in TSC1/TSC2. A Percentage of germline mutation types in TSC1/TSC2. B Distribution of TSC1/TSC2 mutations.

Table 2. Observed frequencies of clinical features for all patients in this study.

\begin{tabular}{|c|c|c|c|}
\hline & $\begin{array}{l}\text { PTC }(-) \\
(N=13)\end{array}$ & $\begin{array}{l}\text { PTC }(+) \\
(N=17)\end{array}$ & $P$-value \\
\hline $\begin{array}{l}\text { Hypomelanotic } \\
\text { macules (\%) }\end{array}$ & $1(7.7)$ & $8(47.1)$ & 0.041 \\
\hline Angiofibromas (\%) & $8(61.5)$ & $12(70.6)$ & 0.705 \\
\hline Ungual fibromas (\%) & $3(23.1)$ & $6(35.3)$ & 0.691 \\
\hline Shagreen patch (\%) & $2(15.4)$ & $3(18.8)$ & 1 \\
\hline $\begin{array}{l}\text { Multiple retinal } \\
\text { hamartomas (\%) }\end{array}$ & $2(15.4)$ & $5(29.4)$ & 0.427 \\
\hline Cortical tuber (\%) & $9(69.2)$ & $17(100)$ & 0.026 \\
\hline SEN (\%) & $9(69.2)$ & $17(100)$ & 0.026 \\
\hline SEGA (\%) & $1(7.7)$ & $3(17.6)$ & 0.613 \\
\hline $\begin{array}{l}\text { Cardiac } \\
\text { rhabdomyoma (\%) }\end{array}$ & $3(23.1)$ & $3(17.6)$ & 1 \\
\hline LAM (\%) & $4(30.8)$ & $7(41.2)$ & 0.708 \\
\hline AML (\%) & $11(84.6)$ & $15(88.2)$ & 1 \\
\hline
\end{tabular}

No difference in AML diameter between patients with and without PTC mutations in TSC2 was observed (Fig. 2B). Regarding the other clinical parameters, a lower age at onset was noted for patients with PTC mutations; however, the differences calculated using the Mann-Whitney U-test were not significant (Fig. 2C). Moreover, a higher incidence of early onset (onset before 20 years old) was observed in patients with PTC mutations than in patients with non-PTC mutations (Fig. 2D). Furthermore, the presence of multiple and bilateral tumors was more frequent in patients with PTC mutations, but the differences were not statistically significant (Fig. 2E, F).

\section{DISCUSSION}

In this study, we performed a genetic analysis of the TSC1 or TSC2 genes in our cohort of 30 patients with TSC. Genetic testing alone is not generally used for diagnostic purposes for patients with TSC, since diagnosis is typically based on clinical findings. If a
Table 3. Observed frequencies of renal clinical features for all patients in this study.

\begin{tabular}{llll} 
& $\begin{array}{l}\text { PTC (-) } \\
(\mathbf{N}=13)\end{array}$ & $\begin{array}{l}\text { PTC (+) } \\
(\mathbf{N}=17)\end{array}$ & P-value \\
\hline $\begin{array}{l}\text { Maximum } \\
\text { diameter of } \\
\text { AML, cm }\end{array}$ & $5.5(2.6-9.0)$ & $3.4(2.0-7.5)$ & 0.722 \\
$\begin{array}{l}\text { Age at onset of } \\
\text { AML, years }\end{array}$ & $27(16-30)$ & $21(14.8-28)$ & 0.621 \\
\hline $\begin{array}{l}\text { Bilateral AML (\%) } \\
\text { Multiple AML (\%) }\end{array}$ & $7(53.8)$ & $11(64.7)$ & 0.711 \\
\hline $\begin{array}{l}\text { Renal cyst (\%) } \\
\text { Bilateral cyst (\%)* }\end{array}$ & $2(15.4)$ & $11(64.7)$ & 1 \\
\hline Multiple cyst (\%)* & $2(100)$ & $7(10)$ & 0.026 \\
\hline
\end{tabular}

*Percent within the cases with renal cysts.

pathological gene mutation is detected, it is a definitive diagnosis. However, if a pathological gene mutation is not detected, no definitive diagnosis can be made ${ }^{17}$. Therefore, genetic diagnosis is not always necessary or useful in renal AML caused by TSC. This is because the genotype-phenotype correlation in TSC is not yet fully understood. Previous studies on TSC genotypes and phenotypes have suggested that the symptoms of patients with TSC2 mutations are more severe than those of patients with TSC1 mutations $s^{5,20-22}$. However, there has been no correlation between mutation types reported.

In previous studies, disease severity was assessed in terms of overall incidence and tumor size $e^{5,13}$. Tumors in TSC have been suggested to be caused by the inactivation of both alleles of either TSC1 or TSC2 genes, consistent with Knudson's two-hit tumor suppressor gene model. Although second-hit mutations are not always observed in each TSC lesion, most tumors in the brain or kidney have second-hit mutations ${ }^{10,23}$. In other words, a second hit is a necessary step for the development of tumors in TSC, and it may be assumed that somatic mutations play a pivotal role in the development of lesions. It is likely that factors other than the type of germline mutation in the TSC1 or TSC2 gene (i.e., modifier genes or environmental factors) may have influenced the incidence or size of tumors. Moreover, the data on the correlation between the type of mutations and the clinical course of the patient might be potentially confounded by whether the mutation was on the TSC1 
A

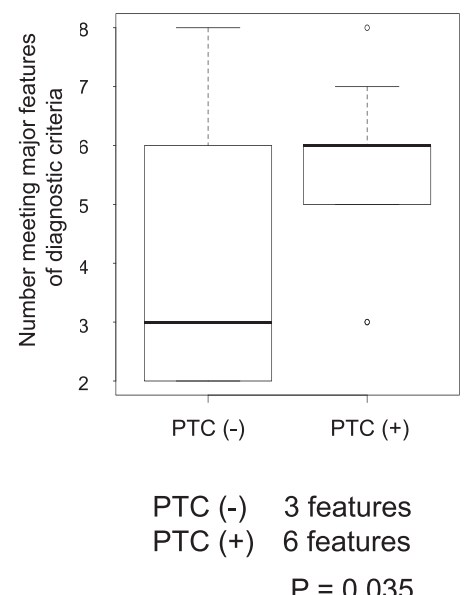

D

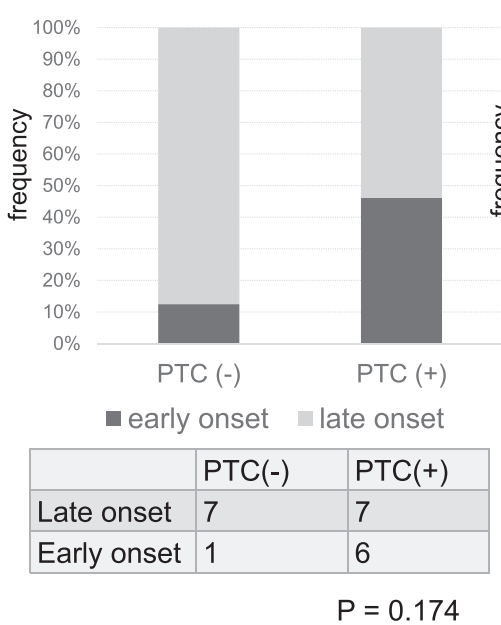

B

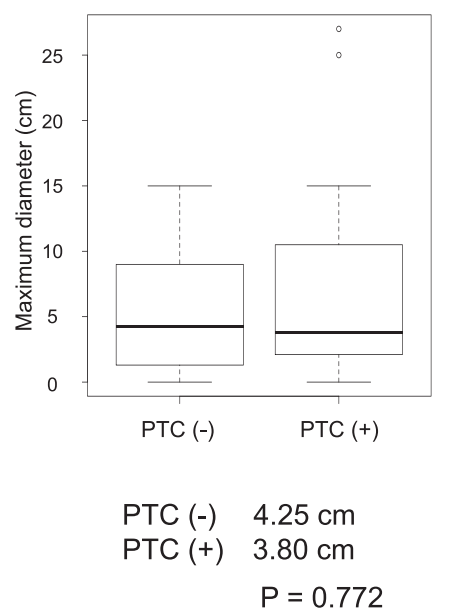

$\mathbf{E}$
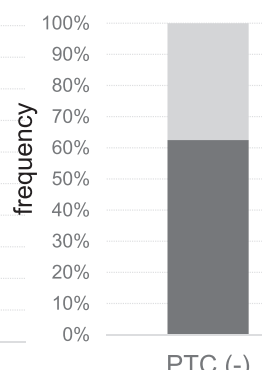

PTC (-)

multiple

\begin{tabular}{|l|l|l|}
\hline & PTC $(-)$ & PTC $(+)$ \\
\hline Single & 3 & 3 \\
\hline Multiple & 5 & 10 \\
\hline
\end{tabular}

C

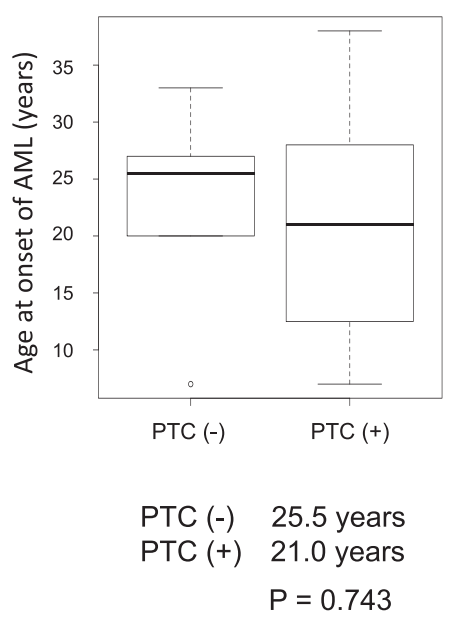

F
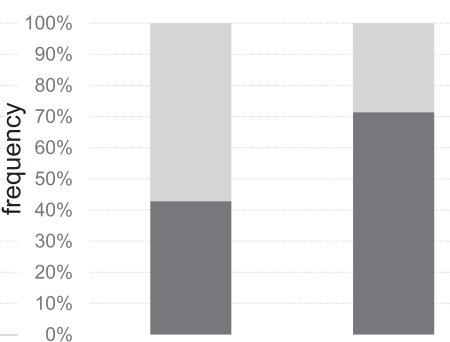

PTC (-)

PTC (+)

a bilateral unilateral

\begin{tabular}{|l|l|l|}
\hline & PTC(-) & PTC $(+)$ \\
\hline Unilateral & 4 & 3 \\
\hline Bilateral & 4 & 10 \\
\hline
\end{tabular}

$P=0.631$

$\mathrm{P}=0.346$

Fig. 2 Phenotype of TSC2. A The number of positive major features listed in the diagnostic criteria ${ }^{18}$. Patients with a PTC were significantly more likely to manifest the major symptoms of the diagnostic criteria than those without a PTC. B There was no significant difference in the maximum diameter of AML with or without a PTC. C Although there was no statistically significant difference in the age of onset of AML between patients with and without a PTC, there was a tendency for patients with a PTC to develop AML at a younger age. D An onset under 20 years of age was defined as early onset, while an onset over 20 years of age was defined as late onset. There was no statistically significant difference, but the percentage of early onset was higher in patients with a PTC. E, F Although not statistically significant, the percentage of multiple and bilateral cases was higher in patients with a PTC.

or TSC2 gene since all the mutations in TSC1 in this study were PTC mutations (Fig. 1).

In this study, we evaluated not only the frequency and diameter of AML but also the age of onset and occurrence of unilateral or bilateral tumors. We presumed that the evaluation of tumor size may have been incorrect in previous studies ${ }^{5,13}$ because tumor diameter is greatly affected by the timing of image evaluation. For this study, tumor diameters were measured based on the first image of the AML diagnosis to align the timing of image evaluation. However, no significant difference in AML diameter was observed between patients with or without PTC mutations. Although the age at onset was lower and the presence of multiple and bilateral tumors was more frequent in patients with PTC mutations, the differences were not statistically significant.

There are two major viewpoints regarding the weak but possible genotype-phenotype correlation. First, despite the effect of the second hit, the majority of second hits in TSC tumors are copy number neutral loss of heterozygosity (CN-LOH). The second viewpoint states that the weak correlation is because of the presence of few missense mutations in the second hit of the tumor $^{10,12}$. Therefore, it is presumed that the residual activity of TSC protein in tumor cells is not affected by the second hit and is dependent on the type of germline mutant allele. If the germline mutation does not contain PTCs, the germline allele will produce tuberin that potentially has partial residual protein activity that may inhibit the mTOR pathway and suppress cell growth. In contrast, tuberin is not produced by NMD in the presence of a PTC. The loss of tuberin activity in cells with mutations with PTCs promotes faster cell growth, which may result in a relatively shorter time between tumor growth and detection. This could potentially result in a lower age of onset and the occurrence of bilateral and multiple tumors.

An alternative idea is that the first hit alone could possibly affect the cellular function responsible for the genotype-phenotype correlation. It is possible that the haploinsufficiency caused by germline mutations with a PTC reduces the amount of tuberin in the cells. This could prevent mTOR inhibition and accelerate the tumor growth of AMLs to a detectable size on imaging at an early stage. In addition, multiple and bilateral AML might be more common in patients with mutations containing a PTC on imaging 
at diagnosis, which is consistent with PTC-promoting tumor growth. Since multiple AMLs that develop in TSC are due to independent second-hit mutations in TSC1 or TSC2 that occur in different progenitor cells ${ }^{12}$, it is possible that a PTC affects the susceptibility of patients to somatic mutations. Other results showed that PTC-containing mutations were significantly more likely to be associated with SEN, cortical tubercles, and renal cysts. A cortical tuber is less likely to have a second hit, suggesting that monoallelic inactivation was sufficient and that the first hit had a strong influence on the phenotype. Our results might suggest that the presence of a PTC in germline mutations alone facilitated the development of some tumors in TSC.

This study has some limitations. The first was the small sample size, which may have been the reason why no statistically significant difference was observed. Since the sample size was small, the inclusion of exceptional cases, such as cases with a rare second hit, might affect the phenotype. This may be another reason for the lack of significant differences. Another limitation was the benign nature of the disease, making it difficult to collect samples from tumor lesions and assess the function of proteins in the lesions. The third limitation was case selection bias owing to the retrospective nature of the study.

In summary, TSC patients with PTC-producing mutations might potentially manifest more severe TSC phenotypes than those with missense mutations. The genetic diagnosis of TSC might be helpful in predicting the severity of TSC disease. A larger-scale study including the functional analysis of the variants deserves further investigation.

\section{REFERENCES}

1. Osborne, J. P., Fryer, A. \& Webb, D. Epidemiology of tuberous sclerosis. Ann. N. Y. Acad. Sci. 615, 125-7 (1991).

2. Crino, P. B., Nathanson, K. L. \& Henske, E. P. The tuberous sclerosis complex. N. Engl. J. Med. 355, 1345-56 (2006).

3. Henske, E. P. et al. Loss of heterozygosity in the tuberous sclerosis (TSC2) region of chromosome band 16p13 occurs in sporadic as well as TSC-associated renal angiomyolipomas. Genes Chromosom. Cancer 13, 295-8 (1995).

4. Shepherd, C. W. et al. Causes of death in patients with tuberous sclerosis. Mayo Clin. Proc. 66(8), 792-6 (1991).

5. Au, K. S. et al. Genotype/phenotype correlation in 325 individuals referred for a diagnosis of tuberous sclerosis complex in the United States. Genet Med. 9, 88-100 (2007).

6. Meng, Y. et al. Mutation landscape of TSC1/TSC2 in Chinese patients with tuberous sclerosis complex. J. Hum. Genet 66, 227-236 (2021).

7. TSC COMPLEX SUBUNIT 2; TSC2. https://www.omim.org/entry/191092 (2020).

8. Tyburczy, M. E. et al. Mosaic and intronic mutations in TSC1/TSC2 explain the majority of TSC patients with no mutation identified by conventional testing. PLoS Genet 11, e1005637 (2015).

9. Knudson, A. G. Jr. Mutation and cancer: statistical study of retinoblastoma. Proc. Natl. Acad. Sci. USA 68, 820-3 (1971).

10. Martin, K. R. et al. The genomic landscape of tuberous sclerosis complex. Nat. Commun. 8, 15816 (2017).

11. Rosset, C. et al. Molecular analysis of TSC1 and TSC2 genes and phenotypic correlations in Brazilian families with tuberous sclerosis. PLoS One 12, e0185713 (2017).

12. Giannikou, K. et al. Whole exome sequencing identifies TSC1/TSC2 biallelic loss as the primary and sufficient driver event for renal angiomyolipoma development. PLoS Genet 12, e1006242 (2016).

13. Li, S. et al. Genotype-phenotype correlation of patients with tuberous sclerosis complex-associated renal angiomyolipoma: a descriptive study. Hum. Pathol. 82, 61-67 (2018).

14. $\mathrm{Ni}$, J. et al. Mutational analysis of renal angiomyolipoma associated with tuberous sclerosis complex and the outcome of short-term everolimus therapy. Sci. Rep. $\mathbf{9}$, 14337 (2019).

15. Kervestin, S. \& Jacobson, A. NMD: a multifaceted response to premature translational termination. Nat. Rev. Mol. Cell Biol. 13, 700-12 (2012).
16. Stefl, S. et al. Molecular mechanisms of disease-causing missense mutations. J. Mol. Biol. 425, 3919-36 (2013).

17. Northrup, H. \& Krueger, D. A., G. International Tuberous Sclerosis Complex Consensus. Tuberous sclerosis complex diagnostic criteria update: recommendations of the 2012 International Tuberous Sclerosis Complex Consensus Conference. Pediatr. Neurol. 49, 243-54 (2013).

18. Richards, S. et al. Standards and guidelines for the interpretation of sequence variants: a joint consensus recommendation of the American College of Medical Genetics and Genomics and the Association for Molecular Pathology. Genet Med. 17, 405-24 (2015).

19. Kanda, Y. Investigation of the freely available easy-to-use software 'EZR' for medical statistics. Bone Marrow Transpl. 48, 452-8 (2013).

20. Jones, A. C. et al. Comprehensive mutation analysis of TSC1 and TSC2-and phenotypic correlations in 150 families with tuberous sclerosis. Am. J. Hum. Genet 64, 1305-15 (1999).

21. Dabora, S. L. et al. Mutational analysis in a cohort of 224 tuberous sclerosis patients indicates increased severity of TSC2, compared with TSC1, disease in multiple organs. Am. J. Hum. Genet 68, 64-80 (2001).

22. Sancak, O. et al. Mutational analysis of the TSC1 and TSC2 genes in a diagnostic setting: genotype-phenotype correlations and comparison of diagnostic DNA techniques in tuberous sclerosis complex. Eur. J. Hum. Genet 13, 731-41. (2005).

23. Bongaarts, A. et al. Subependymal giant cell astrocytomas in tuberous sclerosis complex have consistent TSC1/TSC2 biallelic inactivation, and no BRAF mutations. Oncotarget 8, 95516-95529 (2017).

\section{AUTHOR CONTRIBUTIONS}

Y. M. participated in the design of the study, carried out the molecular biology work, and drafted the manuscript. H. S. and M. S. participated in the design of the study and contributed equally to this work. Y. M., H. I., M. K., T. K., and S. M. carried out the molecular biology work. H. K. and R. S. coordinated and conceived the study and were involved in the critical revision of the manuscript for important intellectual content.

\section{CONFLICT OF INTEREST}

The authors declare no conflicts of interest.

\section{ADDITIONAL INFORMATION}

Supplementary information The online version contains supplementary material available at https://doi.org/10.1038/s41439-022-00181-1.

Correspondence and requests for materials should be addressed to Yoshinari Muto.

Reprints and permission information is available at http://www.nature.com/ reprints

Publisher's note Springer Nature remains neutral with regard to jurisdictional claims in published maps and institutional affiliations.

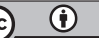

Open Access This article is licensed under a Creative Commons Attribution 4.0 International License, which permits use, sharing, adaptation, distribution and reproduction in any medium or format, as long as you give appropriate credit to the original author(s) and the source, provide a link to the Creative Commons license, and indicate if changes were made. The images or other third party material in this article are included in the article's Creative Commons license, unless indicated otherwise in a credit line to the material. If material is not included in the article's Creative Commons license and your intended use is not permitted by statutory regulation or exceeds the permitted use, you will need to obtain permission directly from the copyright holder. To view a copy of this license, visit http://creativecommons. org/licenses/by/4.0/.

(c) The Author(s) 2022 